\title{
REA_08 - Evaluation of SARS-CoV-2 antigens on a serologic bead-based array assay for diagnostic purposes
}

Juliana Georg da Silva ${ }^{1 *}$; Nara Mazarakis Rubim ${ }^{1}$ Christiane de Fátima Silva Marques ${ }^{1}$.

${ }^{1}$ Fiocruz/Bio-Manguinhos.

Introduction: The SARS-COV-2 pandemic started in Wuhan (China) surprised and forced us to confront our weaknesses as a society and human beings. To face the COVID-19 threat, a worldwide effort was initiated to contain this epidemic in several parts of the world and on different fronts. The diagnostic area is of great importance in the fight against the virus to determine the real dimension of disease distribution in the population and to provide epidemiological estimates for sanitary measures. For this matter, one of the most used techniques is the serological research in which the levels of circulating antibodies are measured in active infection or convalescent individuals, thus assessing the level of immunological memory and protection conferred after the infection.

Objective: In the present study, we used both single and multiplex formats of a bead-based array assay to search for IgG and IgM antibodies for the SARS-CoV-2 virus. By using eight antigens (from either $\mathrm{S}$ and $\mathrm{N}$ proteins) from different suppliers, we intend to investigate the diagnostic feasibility of this technology in the serological screening routine of COVID-19.

Methodology: The test was challenged using 71 sera from an internal laboratory panel to mimic the population serological profile and a synthetic minority oversampling technique (SMOTE) was employed to address the data imbalance problem. For this evaluation, the sensitivity, specificity and accuracy values were determined by machine learning from the median fluorescence intensity (MFI) obtained in each test. The antigens that gathered the best results in the single tests were selected for the multiplex format, in order to verify whether this serological range distinction could be improved.

Results: Preliminary analysis showed that the best conditions were obtained using MES buffer in SF1, S1F2, NF3, NF4 and NF5 antigens; and NaHCO3 buffer for NCF5\#1, NCF5\#2 and NF2. Antigen concentrations of $50 \mu \mathrm{L} / \mathrm{mg}$ and $1 / 200$ sample dilution were the chosen parameters for future analysis due to its achieved performance in all proteins studied. The three antigenic targets that appears to be the most promising were: NF4 for IgG detection, NF2 for IgG and IgM combined detection and NF3 for IgG detection with $98.9 \%, 95.7 \%$ e $94.7 \%$ accuracy, respectively.

Conclusion: These antigens were chosen, together with SF1 and S1F2, in order to better understand the profile and performance of $\mathrm{S}$ and $\mathrm{N}$ proteins in the multiplex assay.

Keywords: bead-based array assay ; diagnosis; SARS-CoV-2 